FACTA UNIVERSITATIS (NIŠ)

Ser. Math. Inform. Vol. 36, No 2 (2021), 309-319

https://doi.org/10.22190/FUMI200708023K

Original Scientific Paper

\title{
SOME RESULT ON LIE IDEALS WITH SYMMETRIC REVERSE BI-DERIVATIONS IN SEMIPRIME RINGS I
}

\author{
Emine Koç Sögütcü and Öznur Gölbaşı \\ Sivas Cumhuriyet University, Faculty of Science, Department of Mathematics, \\ 58140 Sivas, Turkey
}

\begin{abstract}
Let $R$ be a semiprime ring, $U$ a square-closed Lie ideal of $R$ and $D$ : $R \times R \rightarrow R$ a symmetric reverse bi-derivation and $d$ be the trace of $D$. In the present paper, we shall prove that $R$ is commutative ring if any one of the following holds: i) $d(U)=(0)$, ii $d(U) \subset Z$, iii) $[d(x), y] \in Z$, iv $) d(x)$ oy $\in Z$, v) $d([x, y]) \pm[d(x), y] \in Z$, vi $d(x \circ y) \pm(d(x) \circ y) \in Z$, vii $) d([x, y]) \pm d(x) \circ y \in Z \quad$ viii $) d(x \circ y) \pm[d(x), y] \in Z$, ix $) d(x) \circ y \pm[d(y), x] \in Z, \mathrm{x}) d([x, y])-(d(x) \circ y)-[d(y), x] \in Z \mathrm{xi})[d(x), y] \pm[g(y), x] \in Z$, for all $x, y \in U$, where $G: R \times R \rightarrow R$ is symmetric reverse bi-derivation such that $g$ is the trace of $G . z$

Key words: Lie ideals, bi-derivations, actions of Lie algebras
\end{abstract}

\section{Introduction}

Throughout the paper, $R$ will represent an associative ring with center $Z$. A ring $\mathrm{R}$ is said to be prime if $x R y=(0)$ implies that either $x=0$ or $y=0$ and semiprime if $x R x=(0)$ implies that $x=0$, where $x, y \in R$. A prime ring is obviously semiprime. For any $x, y \in R$, the symbol $[x, y]$ stands for the commutator $x y-y x$ and the symbol $x$ oy stands for the commutator $x y+y x$. An additive subgroup $U$ of $R$ is said to be a Lie ideal of $R$ if $[u, r] \in U$, for all $u \in U, r \in R$. $U$ is called a square-closed Lie ideal of $R$ if $U$ is a Lie ideal and $u^{2} \in U$ for all $u \in U$. An additive mapping $d: R \rightarrow R$ is called a derivation if $d(x y)=d(x) y+x d(y)$ holds for all $x, y \in R$. An additive mapping $d: R \rightarrow R$ is said to be a reverse derivation

Received July 08, 2020, accepted January 11, 2021

Communicated by Dijana Mosić

Corresponding Author: Emine Koc Sögütcü, Sivas Cumhuriyet University, Faculty of Science, Department of Mathematics, 58140 Sivas, Turkey | E-mail: eminekoc@cumhuriyet.edu.tr 2010 Mathematics Subject Classification. Primary 16W25; Secondary 16W10, 16 U80.

(C) 2021 by University of Niš, Serbia | Creative Commons License: CC BY-NC-ND 
if $d(x y)=d(y) x+y d(x)$ holds for all $x, y \in R$. A mapping $D(.,):. R \times R \rightarrow R$ is said to be symmetric if $D(x, y)=D(y, x)$ for all $x, y \in R$. A mapping $d: R \rightarrow R$ is called the trace of $D(.,$.$) if d(x)=D(x, x)$ for all $x \in R$.It is obvious that if $D(.,$.$) is$ bi-additive (i.e., additive in both arguments), then the trace $d$ of $D(.,$.$) satisfies the$ identity $d(x+y)=d(x)+d(y)+2 D(x, y)$, for all $x, y \in R$. If $D(.,$.$) is bi-additive$ and satisfies the identities

$$
D(x y, z)=D(x, z) y+x D(y, z)
$$

and

$$
D(x, y z)=D(x, y) z+y D(x, z),
$$

for all $x, y, z \in R$. Then $D(.,$.$) is called a symmetric bi-derivation. If D(.,$.$) is$ reverse bi-additive and satisfies the identity

$$
D(x y, z)=D(y, z) x+y D(x, z)
$$

and

$$
D(x, y z)=D(x, z) y+z D(x, y) .
$$

Then $D(.,$.$) is called a symmetric reverse bi-derivation.$

The study of commuting mappings was initiaded by a well-known theorem due to Posner [7] which stetes that the existence of a nonzero commuting derivation on a prime ring $R$ implies that $R$ is commutative. A number of authors have extended the Posner's theorem in several ways. The notion of additive commuting mapping is closely connected with the notion of bi-derivation. Every additive commuting mapping $F: R \rightarrow R$ gives rise to a bi-derivation on $R$. Namely, linearizing $[F(x), x]=0$, we get $[F(x), y]=[x, F(y)]$ and we note that the map $(x, y) \longmapsto[F(x), y]$ is a biderivation. The concept of bi-derivation was introduced by Maksa in [5]. It is shown in [6] that symmetric bi-derivations are related to general solution of some functional equations. Some results concerning symmetric bi-derivations in prime rings can found in [9] and [10].

In [4], Herstein showed that if $R$ is a prime ring of characteristic different from two and $d$ is a nonzero derivation such that $d(R) \subset Z$, then $R$ must be commutative. Bergen et al. proved the following results in [2]: Let $R$ be a prime ring of characteristic different from $2, U$ a nonzero Lie ideal of $R$ and $d$ a nonzero derivation. If $d(U) \subset Z$, then $U \subset Z$. Several authors investigated this result for a prime ring admitting derivation or generalized derivation.

Many authors investigated the commutativity of prime or semiprime rings satisfying certain functional identities involving derivation or generalized derivation. In this paper, we extend some well known these results concerning of Lie ideals in semiprime rings to a reverse bi-derivations. Throughout the present paper, we shall make use of the following basic identities without any specific mentioning:

i) $[x, y z]=y[x, z]+[x, y] z$

ii) $[x y, z]=[x, z] y+x[y, z]$

iii) $x y o z=(x \circ z) y+x[y, z]=x(y o z)-[x, z] y$

iv) $x o y z=y(x o z)+[x, y] z=(x o y) z+y[z, x]$. 


\subsection{Results}

Lemma 1.1. [1, Theorem 1.3] Let $R$ be a 2 - torsion free semiprime ring and $U$ a noncentral Lie ideal of $R$ such that $u^{2} \in U$ for all $x \in U$. Then there exists a nonzero ideal $I$ of $R$ such that $I \subseteq U$.

Lemma 1.2. [3, Lemma $2(b)]$ If $R$ is a semiprime ring, then the center of a nonzero ideal of $R$ is contained in the center of $R$.

Lemma 1.3. [8, Theorem 2.1] Let $R$ be a semiprime ring, $I$ a nonzero two-sided ideal of $R$ and $a \in R$ such that axa $=0$ for all $x \in I$, then $a=0$.

Lemma 1.4. Let $R$ be a semiprime ring. If a nonzero ideal of $R$ is in the center of $R$, then $R$ is a commutative ring.

Proof. By the hypothesis, we get

$$
[x, r]=0, \text { for all } x \in I, r \in R .
$$

Replacing $x$ by $s x, s \in R$ in this equation and using this equation, we obtain that

$$
[s, r] x=0, \text { for all } x \in I, r \in R .
$$

Thus, $[R, R] I=(0)$. Multiplying this equation on the right by $[R, R]$, we have $[R, R] I[R, R]=(0)$. By Lemma 3 , we conclude that $R$ is a commutative ring. The proof is completed.

Theorem 1.1. Let $R$ be a 2-torsion free semiprime ring, $U$ a square-closed Lie ideal of $R$ and $D: R \times R \rightarrow R$ a symmetric reverse bi-derivation and $d$ be the trace of $D$. If $d(U)=(0)$, then $D=0$.

Proof. By Lemma 1, there exists a nonzero ideal $I$ of $R$ such that $I \subseteq U$. By the hypothesis, we have

$$
d(x)=0, \text { for all } x \in I .
$$

Replacing $x$ by $x+y, y \in I$ in this equation and using the hypothesis, we get

$$
2 D(x, y)=0, \text { for all } x, y \in I .
$$

Since $R$ is 2 -torsion free, we have

$$
D(x, y)=0, \text { for all } x, y \in I .
$$

Taking $x$ by $x r, r \in R$ in the above equation and using this equation, we obtain that

$$
D(r, y) x=0, \text { for all } x, y \in I, r \in R \text {. }
$$


Replacing $y$ by $y s, s \in R$, we have

$$
D(r, s) y x=0, \text { for all } x, y \in I, r, s \in R .
$$

Multiplying this equation on the right by $D(r, s) y$, we get

$$
D(r, s) y I D(r, s) y=0, \text { for all } y \in I, r, s \in R .
$$

By Lemma 3, we arrive at

$$
D(r, s) y=0, \text { for all } y \in I, r, s \in R \text {. }
$$

Again, multiplying this equation on the right by $D(r, s)$, we find that

$$
D(r, s) y D(r, s)=0, \text { for all } y \in I, r, s \in R .
$$

Using Lemma 3 in the above equation, we get $D=0$. The proof is completed.

Theorem 1.2. Let $R$ be a 2-torsion free semiprime ring, $U$ a square-closed Lie ideal of $R$ and $D: R \times R \rightarrow R$ a symmetric reverse bi-derivation and $d$ be the trace of $D$. If $d(U) \subset Z$, then $d$ is commuting on $I$ where $I$ is nonzero ideal of $R$.

Proof. By Lemma 1, there exists a nonzero ideal $I$ of $R$ such that $I \subseteq U$. By our hypothesis, we get

$$
d(x) \in Z, \text { for all } x \in I .
$$

Replacing $x$ by $x+y, y \in I$ in above expression, we get

$$
d(x)+d(y)+2 D(x, y) \in Z, \text { for all } x, y \in I .
$$

Using the hypothesis and $R$ is 2 -torsion free, we have

$$
D(x, y) \in Z \text {, for all } x, y \in I \text {. }
$$

Commuting this term with $r, r \in R$, we get

$$
[D(x, y), r]=0, \text { for all } x, y \in I, r \in R \text {. }
$$

Taking $x$ by $x s, s \in R$ in the last equation, we obtain that

$$
[s D(x, y)+D(s, y) x, r]=0, \text { for all } x, y \in I, r, s \in R .
$$

Using equation (2.1), we get

$$
[s, r] D(x, y)+D(s, y)[x, r]+[D(s, y), r] x=0 \text {, for all } x, y \in I, r, s \in R \text {. }
$$

Replacing $s$ by $x$ in the last equation, we get

$$
[x, r] D(x, y)+D(x, y)[x, r]+[D(x, y), r] x=0, \text { for all } x, y \in I, r, s \in R \text {. }
$$


Applying equation (2.1), we see that

$$
2[x, r] D(x, y)=0, \text { for all } x, y \in I, r \in R .
$$

Since $R$ is 2 -torsion free, we get

$$
[x, r] D(x, y)=0, \text { for all } x, y \in I, r \in R .
$$

Using $D(x, y) \in Z$, we have

$$
[x, r] t D(x, y)=0, \text { for all } x, y \in I, r, t \in R .
$$

Taking $y$ by $x$, we have

$$
[x, r] t d(x)=0, \text { for all } x, \in I, r, t \in R .
$$

Replacing $r$ by $d(x)$ in this equation, we find that

$$
[x, d(x)] t d(x)=0, \text { for all } x, \in I, t \in R .
$$

Multiplying this equation on the right by $x$, we get

$$
[x, d(x)] t d(x) x=0, \text { for all } x, \in I, t \in R .
$$

Taking $t$ by $t x$ in equation (2.2), we find that

$$
[x, d(x)] \operatorname{txd}(x)=0, \text { for all } x, \in I, t \in R .
$$

Subtracting two last equations, we arrive at

$$
[x, d(x)] t[x, d(x)]=0 \text {, for all } x, \in I, t \in R .
$$

Since $R$ is semiprime ring, we obtain that $d$ is commuting on $I$. The proof is completed.

Theorem 1.3. Let $R$ be a 2-torsion free semiprime ring, $U$ a square-closed Lie ideal of $R$ and $D: R \times R \rightarrow R$ a symmetric reverse bi-derivation and $d$ be the trace of $D$. If $[d(x), y] \in Z$, for all $x, y \in U$, then $d$ is commuting on I where $I$ is nonzero ideal of $R$.

Proof. By Lemma 1, there exists a nonzero ideal $I$ of $R$ such that $I \subseteq U$. By the hypothesis, we get

$$
[d(x), y] \in Z, \text { for all } x, y \in I .
$$

Replacing $y$ by $y z$ in the hypothesis, we have

$$
[d(x), y] z+y[d(x), z] \in Z, \text { for all } x, y, z \in I .
$$

Commuting this term with $r, r \in R$, we get

$$
[[d(x), y] z+y[d(x), z], r]=0,
$$


and so,

$$
[d(x), y][z, r]+[y, r][d(x), z]=0 \text { for all } x, y, z \in I, r \in R .
$$

Replacing $r$ by $z$ in the last equation, we obtain that

$$
[y, z][d(x), z]=0, \text { for all } x, y, z \in I .
$$

Taking $y$ by $t y, t \in R$ in above equation, we see that

$$
[t, z] y[d(x), z]=0 \text { for all } x, y, z \in I, r \in R \text {. }
$$

Replacing $t$ by $d(x)$, we get

$$
[d(x), z] y[d(x), z]=0, \text { for all } x, y, z \in I .
$$

By Lemma 3, we have

$$
[d(x), z]=0, \text { for all } x, z \in I \text {. }
$$

Using Lemma 2, we obtain that $d(x) \in Z$, for all $x \in I$. We conclude that $d$ is commuting on $I$ by Theorem 2 .

Theorem 1.4. Let $R$ be a 2-torsion free semiprime ring, $U$ a square-closed Lie ideal of $R$ and $D: R \times R \rightarrow R$ a symmetric reverse bi-derivation and $d$ be the trace of $D$. If $d(x) \circ y \in Z$, for all $x, y \in U$, then $d$ is commuting on $I$ where $I$ is nonzero ideal of $R$.

Proof. By Lemma 1, there exists a nonzero ideal $I$ of $R$ such that $I \subseteq U$. By the hypothesis, we get

$$
d(x) \circ y \in Z \text {, for all } x, y \in I .
$$

Taking $y$ by $y z$ in the last expression, we obtain that

$$
y(d(x) \circ z)+[d(x), y] z \in Z \text {, for all } x, y, z \in I .
$$

Commuting this term with $r, r \in R$, we see that

(1.3) $[d(x), y][z, r]+[y, r](d(x) \circ z)+[[d(x), y], r] z=0$, for all $x, y, z \in I, r \in R$.

Taking $z$ by $z t, t \in R$ in the above equation, we get

$[d(x), y][z, r] t+[d(x), y] z[t, r]+[y, r](d(x) \circ z) t+[y, r] z[t, d(x)]+[[d(x), y], r] z t=0$.

Using equation (2.3), we get

$$
[d(x), y] z[t, r]+[y, r] z[t, d(x)]=0, \text { for all } x, y, z \in I, r, t \in R .
$$

Replacing $t$ by $d(x)$ and $r$ by $y$, we have

$$
[d(x), y] z[d(x), y]=0, \text { for all } x, y, z \in I .
$$

By Lemma 3, we have

$$
[d(x), z]=0, \text { for all } x, z \in I .
$$

We conclude that $d$ is commuting on $I$ by Theorem 2 and Lemma 2 . 
Theorem 1.5. Let $R$ be a 2-torsion free semiprime ring, $U$ a square-closed Lie ideal of $R$ and $D: R \times R \rightarrow R$ a symmetric reverse bi-derivation and $d$ be the trace of $D$. If $d([x, y]) \pm[d(x), y] \in Z$, for all $x, y \in U$, then $d$ is commuting on $I$ where $I$ is nonzero ideal of $R$.

Proof. By Lemma 1, there exists a nonzero ideal $I$ of $R$ such that $I \subseteq U$. By the hypothesis, we have

$$
d([x, y]) \pm[d(x), y] \in Z, \text { for all } x, y \in I
$$

Writting $y$ by $y+z, z \in I$, we have

$$
d([x, y])+d([x, z])+2 D([x, y],[x, z]) \pm[d(x), y] \pm[d(x), z] \in Z
$$

By the hypothesis, we get

$$
2 D([x, y],[x, z]) \in Z .
$$

Since $R$ is 2 -torsion free, we see that

$$
D([x, y],[x, z]) \in Z, \text { for all } x, y \in I .
$$

Replacing $y$ by $z$ in the last expression, we get

$$
D([x, y],[x, y]) \in Z \text {, for all } x, y \in I .
$$

That is,

$$
d([x, y]) \in Z, \text { for all } x, y \in I .
$$

By the hypothesis, we have

$$
[d(x), y] \in Z, \text { for all } x, y \in I .
$$

By Theorem 3, we conclude that $d$ is commuting on $I$.

Theorem 1.6. Let $R$ be a 2-torsion free semiprime ring, $U$ a square-closed Lie ideal of $R$ and $D: R \times R \rightarrow R$ a symmetric reverse bi-derivation and $d$ be the trace of $D$. If $d(x \circ y) \pm(d(x) \circ y) \in Z$, for all $x, y \in U$, then $d$ is commuting on $I$ where $I$ is nonzero ideal of $R$.

Proof. By Lemma 1, there exists a nonzero ideal $I$ of $R$ such that $I \subseteq U$. We have

$$
d(x \circ y) \pm(d(x) \circ y) \in Z, \text { for all } x, y \in I .
$$

Taking $y$ by $y+z, z \in I$, we get

$$
d(x \circ y)+d(x \circ z)+2 D(x \circ y, x \circ z) \pm d(x) \circ y \pm d(x) \circ z \in Z
$$

By the hypothesis and since $R$ is 2-torsion free, we have

$$
D(x \circ y, x \circ z) \in Z \text {. }
$$


Replacing $y$ by $z$ in the this expression, we get

$$
D(x \circ y, x \circ y) \in Z, \text { for all } x, y \in I,
$$

and so,

$$
d(x \circ y) \in Z, \text { for all } x, y \in I .
$$

Using the hypothesis, we obtain that

$$
d(x) \circ y \in Z, \text { for all } x, y \in I .
$$

We see that $d$ is commuting on $I$ by Theorem 4 .

Theorem 1.7. Let $R$ be a 2 -torsion free semiprime ring, $U$ a square-closed Lie ideal of $R$ and $D: R \times R \rightarrow R$ a symmetric reverse bi-derivation and $d$ be the trace of $D$. If $d([x, y]) \pm d(x) \circ y \in Z$, for all $x, y \in U$, then $d$ is commuting on $I$ where $I$ is nonzero ideal of $R$.

Proof. By Lemma 1, there exists a nonzero ideal $I$ of $R$ such that $I \subseteq U$. We have

$$
d([x, y]) \pm(d(x) \circ y) \in Z, \text { for all } x, y \in I .
$$

Taking $y$ by $y+z, z \in I$ in the hypothesis, we get

$$
d([x, y])+d([x, z])+2 D([x, y],[x, z]) \pm d(x) \circ y \pm d(x) \circ z \in Z
$$

Using the hypothesis and since $R$ is 2 -torsion free, we find that

$$
D([x, y],[x, z]) \in Z \text {. }
$$

Writting $y$ by $z$ in the above expression, we see that

$$
D([x, y],[x, y]) \in Z \text {, for all } x, y \in I .
$$

That is,

$$
d([x, y]) \in Z, \text { for all } x, y \in I .
$$

By the hypothesis, we have

$$
d(x) \circ y \in Z, \text { for all } x, y \in I \text {. }
$$

By Theorem 4, we obtain that $d$ is commuting on $I$.

Theorem 1.8. Let $R$ be a 2 -torsion free semiprime ring, $U$ a square-closed Lie ideal of $R$ and $D: R \times R \rightarrow R$ a symmetric reverse bi-derivation and $d$ be the trace of $D$. If $d(x \circ y) \pm[d(x), y] \in Z$, for all $x, y \in U$, then $d$ is commuting on $I$ where $I$ is nonzero ideal of $R$. 
Proof. By Lemma 1, there exists a nonzero ideal $I$ of $R$ such that $I \subseteq U$. We get

$$
d(x \circ y) \pm[d(x), y] \in Z, \text { for all } x, y \in I .
$$

Replacing $y$ by $y+z, z \in I$, we get

$$
d(x \circ y)+d(x \circ z)+2 D(x \circ y, x \circ z) \pm[d(x), y] \pm[d(x), z] \in Z .
$$

Using the hypothesis and $R$ is 2 -torsion free, we have

$$
D(x \circ y, x \circ z) \in Z .
$$

Writting $y$ by $z$ in the last expression, we get

$$
D(x \circ y, x \circ y) \in Z \text {, for all } x, y \in I,
$$

and so, $d(x \circ y) \in Z$, for all $x, y \in I$. Using the hypothesis, we have

$$
[d(x), y] \in Z, \text { for all } x, y \in I .
$$

We conclude that $d$ is commuting on $I$ by Theorem 3 .

Theorem 1.9. Let $R$ be a 2-torsion free semiprime ring, $U$ a square-closed Lie ideal of $R$ and $D: R \times R \rightarrow R$ a symmetric reverse bi-derivation and $d$ be the trace of $D$. If $d(x) \circ y \pm[d(y), x] \in Z$, for all $x, y \in U$, then $d$ is commuting on I where $I$ is nonzero ideal of $R$.

Proof. By Lemma 1, there exists a nonzero ideal $I$ of $R$ such that $I \subseteq U$. We have

$$
d(x) \circ y \pm[d(y), x] \in Z, \quad \text { for all } x, y \in I .
$$

Replacing $y$ by $y+z, z \in I$, we get

$$
d(x) \circ y+d(x) \circ z \pm 2[D(y, z), x] \pm[d(y), x] \pm[d(z), x] \in Z
$$

Applying the hypothesis, we see that

$$
2[D(y, z), x] \in Z .
$$

Since $R$ is $2-$ torsion free, we find that

$$
[D(y, z), x] \in Z .
$$

Replacing $z$ by $y$ in this expression, we get

$$
[D(y, y), x] \in Z \text {, for all } x, y \in I
$$

and so, $[d(y), x] \in Z$, for all $x, y \in I$. Using the hypothesis, we have

$$
d(x) \circ y \in Z, \text { for all } x, y \in I .
$$

By Theorem 4, we conclude that $d$ is commuting on $I$. 
Theorem 1.10. Let $R$ be a 2 -torsion free semiprime ring, $U$ a square-closed Lie ideal of $R$ and $D: R \times R \rightarrow R, G: R \times R \rightarrow R$ two symmetric reverse bi-derivations where $d$ is the trace of $D$ and $g$ is the trace of $G$. If $[d(x), y] \pm[g(y), x] \in Z$, for all $x, y \in U$, then $d$ is commuting on $I$ where $I$ is nonzero ideal of $R$.

Proof. By Lemma 1, there exists a nonzero ideal $I$ of $R$ such that $I \subseteq U$. We get

$$
[d(x), y] \pm[g(y), x] \in Z, \text { for all } x, y \in I
$$

Taking $y$ by $y+z, z \in I$, we get

$$
[d(x), y]+[d(x), z] \pm[g(y), x] \pm[g(z), x] \pm 2[G(y, z), x] \in Z
$$

Since $R$ is 2 -torsion free and using the hypothesis, we obtain

$$
[G(y, z), x] \in Z \text {. }
$$

Replacing $y$ by $z$ in the above expression, we have

$$
[G(y, y), x] \in Z \text {, for all } x, y \in I \text {. }
$$

That is,

$$
[g(y), x] \in Z, \text { for all } x, y \in I .
$$

We see that $d$ is commuting on $I$ by Theorem 3 .

Theorem 1.11. Let $R$ be a 2 -torsion free semiprime ring, $U$ a square-closed Lie ideal of $R$ and $D: R \times R \rightarrow R$ a symmetric reverse bi-derivation and $d$ be the trace of D. If $d([x, y])-(d(x) \circ y)-[d(y), x] \in Z$, for all $x, y \in U$, then $d$ is commuting on $I$ where $I$ is nonzero ideal of $R$.

Proof. By Lemma 1, there exists a nonzero ideal $I$ of $R$ such that $I \subseteq U$. By the hypothesis, we obtain that

$$
d([x, y])-(d(x) \circ y)-[d(y), x] \in Z, \text { for all } x, y \in I .
$$

Taking $y$ by $y+z, z \in I$, we get

$$
\begin{aligned}
d([x, y]) & +d([x, z])+2 D([x, y],[x, z]) \\
& -d(x) \circ y-d(x) \circ z-[d(y), x]-[d(z), x]-2[D(y, z), x] \in Z .
\end{aligned}
$$

Using the hypothesis, we have

$$
D([x, y],[x, z])-[D(y, z), x] \in Z .
$$

Replacing $y$ by $z$ in this expression, we see that

$$
D([x, y],[x, y])-[D(y, y), x] \in Z \text {, for all } x, y \in I .
$$


That is,

$$
d([x, y])-[d(y), x] \in Z, \text { for all } x, y \in I .
$$

Hence we can write

$$
d([x, y])-[d(y), x]-d(y) \circ x+d(y) \circ x \in Z
$$

and using the hypothesis, we get

$$
d(y) \circ x \in Z, \text { for all } x, y \in I .
$$

By Theorem 4, we conclude that $d$ is commuting on $I$.

\section{REF E R E N C E S}

1. Z. Bedir and O. Golbasi: Some commutativity on Lie ideals on semiprime rings. Adiyaman University Journal of Science, 10(2) (2020), 548-556.

2. J. Bergen, I. N. Herstein and J. W. KerR: Lie ideals and derivations of prime rings. J. Algebra, 71(1) (1981), 259-267.

3. M. N. DAIF and H. E. BelL: Remarks on derivations on semiprime rings. Internat J. Math. Math. Sci. 15(1) (1992) 205-206.

4. I. N. Herstein: A note on derivations. Canad. Math. Bull. 21(3) (1978), 369-370.

5. Gy. Maksa: $A$ remark on symmetric biadditive functions having non-negative diagonalization. Glasnik. Mat. 15(35) (1980), 279-282.

6. Gy. Maksa: On the trace of symmetric biderivations. C. R. Math. Rep. Acad. Sci. Canada, 9 (1987), 303-307.

7. E. C. Posner: Derivations in prime rings. Proc. Amer. Soc. 8 (1957), 1093-1100.

8. M. S. Samman and A.B. Thaheem: Derivations on semiprime rings. Int. J. Pure Appl. Math. 5(4) (2003), 465-472.

9. J. VuKman: Symmetric biderivations on prime and semiprime rings. Aequationes Math. 38 (1989), 245-254.

10. J. VuKman: Two results concerning symmetric biderivations on prime rings. Aequationes Math. 40 (1990), 181-189. 